\title{
GRAIL月球重力场模型对嫦娥卫星定轨精度的 改进
}

\author{
段建锋 $1,2^{*}$, 曹建峰 ${ }^{1,3}$, 陈明 ${ }^{1}$, 张宇 ${ }^{1}$ \\ 1. 北京航天指挥控制中心, 北京 100094 ; \\ 2. 清华大学航天航空学院, 北京 100083 ; \\ 3. 航天飞行动力学技术重点实验室, 北京 100094 \\ *联系人, E-mail: duanjf1987@sina.com
}

收稿日期: 2016-07-04; 接受日期: 2016-09-19; 网络出版日期: 2017-01-24

\begin{abstract}
摘要从阶方差以及大地水准面自由空气重力异常两个方面分析了“重力恢复与内部实验室”(Gravity Recovery and Interior Laboratory, GRAIL) 月球重力场420阶模型(GL0420A)的特点, 并通过与月球勘探者(Lunar Prospector, LP) 重力场 165 阶模型(LP165P)分析结果比较发现, 该重力场极大地提高了月球重力场的阶数及误差精度, 在全 月面都有明显的重力异常正负交替现象. 本文使用 GL0420A重力场对“嫦娥”系列探测器的绕月测轨数据进行 轨道解算, 对比LP165P重力场模型解算结果, 在定轨弧段内有 $10 \mathrm{~m}$ 量级的精度提升, 可将该重力场用于高精度 的月球探测器精密定轨。
\end{abstract}

关键词“嫦娥”探测器, GL0420A月球重力场模型, LP165P月球重力场模型, 精度分析

PACS: 95.55.Pe, 91.10.Fc, 95.40.+s, 91.10.Op

\section{1 引言}

月球重力场的研究是月球科学的一个重要部分, 是研究月球物理性质及内部结构、月球起源和演化 等科学问题的主要手段 ${ }^{[1]}$, 高精度月球重力场模型给 出的月球主转动惯量可以约束月球内核的大小及状 态, 弥补月震观测的不足对月核研究带来的限制或缺 陷 ${ }^{[2]}$. 自 1966年前苏联发射了月球探测器 10 (Luna10) 后, 人类就开始了月球重力场的研究, 对月球重力场 的高精度探测研究具有重要的理论意义和应用价值, 至今仍然是月球探测的关键问题之一, 高精度的月球
重力场对于月球探测, 尤其是低轨月球探测的轨道支 持非常重要 ${ }^{[3]}$.

早期的月球重力场模型都存在相同的问题, 即由 于月球的公转、自转轨道周期相同, 月球的背面始终 背对地球, 基于常规的地基测量无法直接获取月球背 面的测轨数据, 月球背面的重力异常只能通过拟合间 接求出, 这极大地制约了月球重力场模型的精度 ${ }^{[4]}$.

LP (Lunar Prospector)是美国宇航局(National Aeronautics and Space Administration, NASA)实施的探索计 划中的第 3 个发射任务, 探索计划的主要目的是开发 频繁的、低成本的太阳系探测任务. 最初的设想是

引用格式: 段建锋, 曹建峰, 陈明, 等. GRAIL月球重力场模型对嫦娥卫星定轨精度的改进. 中国科学: 物理学 力学天文学, 2017, 47: 069502 Duan J F, Cao J F, Chen M, et al. The improvements of Chang'E series satellites orbit determination from GRAIL lunar gravity model (in Chinese). Sci Sin-Phys Mech Astron, 2017, 47: 069502, doi: 10.1360/SSPMA2016-00320 
通过中继方式获取月球背面的直接观测数据以提高 月球背面重力场的分辨率, 后来因为经费原因而被取 消 ${ }^{[5,6]}$, 只能采用单颗卫星观测的方式, 获得月球正面 的重力场信息.

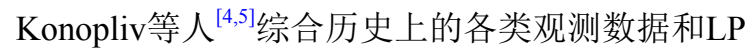
的多普勒及测距测量数据, 研制了150阶次的重力场 模型(LP150Q)和165阶次的重力场模型LP165P. 虽然 LP165P模型的阶次达到了 165, 但由于缺少远月面数 据的支持, 谐系数之间存在很强的相关性, 使得其在 近月面的有效阶次为 110 阶次, 远月面仅为 60 阶次 ${ }^{[5]}$. 在实际应用中, 相比其他重力场模型, 该模型表现出 较好的轨道预报精度, 与月球地形及质量瘤的分布也 有更好的相关性 ${ }^{[7]}$.

于 2002年3月17日发射升空的“重力恢复和气候试 验”(Gravity Recovery and Climate Experiment, GRACE) 双星计划是美国和德国共同研制开发的, 成功运用了 卫星高低/低低测量模式(Satellite to Satellite Tracking in High-Low/Low-Low, SSTHL/LL) 以及高精度、高空间 分辨率地球重力场静态及时变探测方法, 进而促进了 大地测量学、固体地球物理学、海洋学、地震学、空 间科学、天文学、国防建设等领域的快速发展 ${ }^{[8]}$. 基 于GRACE的优秀表现, 美国NASA于 2011年9月10日发 射了 GRAIL (Gravity Recovery and Interior Laboratory) 月球探测重力双星, GRAIL探月计划是美国NASA实施 的“未来太空探索计划”的核心部分 ${ }^{[9]}$. GRAIL计划包含 两个相同的月球探测器, 分别为GRAIL-A和GRAIL-B, 它们运行在 $50 \mathrm{~km}$ 的近圆形月球轨道上, 利用 $\mathrm{Ka}$ 频段 在两个探测器之间进行高精度距离变化率测量, 通 过对这些测量数据的精确分析, Zuber等人 ${ }^{[10]}$ 获取了 高精度的GRAIL月球重力场 GL0420A, GRGM540A, GRGM660PRIM, GL0660B, GRGM900C, GL0900D等 模型, 极大地提高了月球重力场的阶数 ${ }^{[1-14]}$.

长期以来, 我国的探月工程任务一直使用LP165P 月球重力场模型进行相关计算分析, 缺乏高精度的 月球重力场模型 ${ }^{[15]}$. 在未来对轨道精度要求更高的 月球交会对接任务中, 高精度月球重力场模型的应 用显得无为重要. 在现有的测量数据精度下, 对比 GRGM660PRIM, GL0900D, GL420A等模型的计算精 度, 本文作者在实际计算过程中发现模型阶数越高, 计 算效率越慢, 计算时间呈倍数上升,而所获得的定轨 精度几乎没有提高, 文献[16,17]中对此进行了更为详
细的分析, 因此本文结合对月球重力场模型LP165P和 GL0420A的特点、相互之间差异的分析, 并利用“嫦 娥” 系列探测器 (嫦娥一号、嫦娥二号) 的测轨数据使用 GL0420A, LP165P模型进行处理, 分析该模型所具备 的轨道计算、预报能力. 轨道计算分析采用的是北京 航天飞行控制中心的轨道计算与分析软件平台(BACC Orbit Determination and Analysis System, BODAS ${ }^{[18]}$.

\section{2 重力场模型分析}

\section{1 重力异常比较}

通常为了描述天体外部的重力位, 人为地选择一 个形状规则的自转质体作为近似. 该质体满足两个 条件: 外部的重力场计量与实际外部的重力场接近; 表面为自身重力位的等值面, 且尽量与大地水准面接 近 ${ }^{[19]}$. 对于月球通常选取半径为 $1738 \mathrm{~km}$ 的参考球.

为了评价重力场模型的情况, 通常采用大地水准 面的自由空气重力异常分布来描述. 月球自由空气重 力异常的计算公式为 ${ }^{[20]}$

$$
\begin{aligned}
\Delta g= & \frac{G M}{R^{2}} \sum_{l=2}^{n} \sum_{m=0}^{l}\left(\frac{R}{r}\right)^{l}(l-1) \bar{P}_{l m}(\sin \varphi) \\
& \times\left(\bar{C}_{l m} \cos m \lambda+\bar{S}_{l m} \sin m \lambda\right),
\end{aligned}
$$

其中 $G M$ 为月球的月心引力常数, $R$ 为月球重力场模型 使用的参考半径, $r$ 为距离月心的距离, $\bar{P}_{l m}$ 为完全归一 化的缔合勒让德多项式, $\bar{C}_{l m}, \bar{S}_{l m}$ 为 $n$ 阶 $m$ 次完全正则化 的重力场位系数, $\lambda$ 为月球固连坐标系经度, $\varphi$ 为纬度.

LP165P和GL0420A月球重力场模型使用了相同 的参考半径, 均为 $1738 \mathrm{~km}$, 但是采用的引力常数不 相同. 图1为两个重力场模型自由空气重力异常的分 布, 图1(a)是基于LP165P重力场模型, 截断至165阶次 位系数计算得到的月球表面(1738 km高度的正球)自 由空气重力异常分布图, 图1(b)是基于GL0420A重力 场模型, 截断至420阶次位系数计算得到的月球表面 (1738 km高度的正球)自由空气重力异常分布图. 图 形均使用赫墨尔等面积投影方式(Hammer Equal-Area Projections), 图中左边为远月面(Far-Side), 右边为近月 面(Near-Side). 单位采用毫伽(mGAL). 由图1可以发现, 两个模型在近月面自由空气重力异常的分布相近, 而 在远月面存在较大差异; GL0420A能明显地揭示远月 面典型区域自由空气重力异常的特征, 即负的重力异 
常包围正的重力异常, 外层又由正的重力异常包围, 出现正负重力异常交替的现象, LP165P对这一特征则 显得很模糊. 另外, GL0420A 能够明显地分辨出远月 面小型撞击盆地的自由空气重力异常, 而LP165P模型 则无法分辨出这一特征.

\section{2 重力场模型谱分析比较}

重力场解算中, 高阶重力场位系数的确定通常借 助于Kaula约束, 即Kaula准则

$\mathrm{RMS}_{n}=\frac{K}{n^{2}}$,

式中 $K$ 为常数, $n$ 为阶数. 该准则也常用于检验一个重 力场模型的可靠性, 即正常的位系数具有零均值, 其 阶方差与阶数 $n$ 的平方成反比. 重力场模型的功率谱 常用阶方差来描述, 其计算公式如下 ${ }^{[21]}$ :

$\sigma_{n}=\sqrt{\frac{\sum_{m=0}^{n}\left(\bar{C}_{n m}^{2}+\bar{S}_{n m}^{2}\right)}{2 n+1}}$,

式中 $\bar{C}_{n m}$ 与 $\bar{S}_{n m}$ 表示 $n$ 阶 $m$ 次完全正则化的位系数, 用以 表示重力场在频率域中的强度, 误差阶方差则可以反 映重力场的误差.

图 2 是对数坐标图, 描述的是月球重力场模型 GL0420A, LP165P位系数的阶方差及误差阶方差. 图 中点虚线表示Kaula约束曲线, 叉型符号表示GL0420A
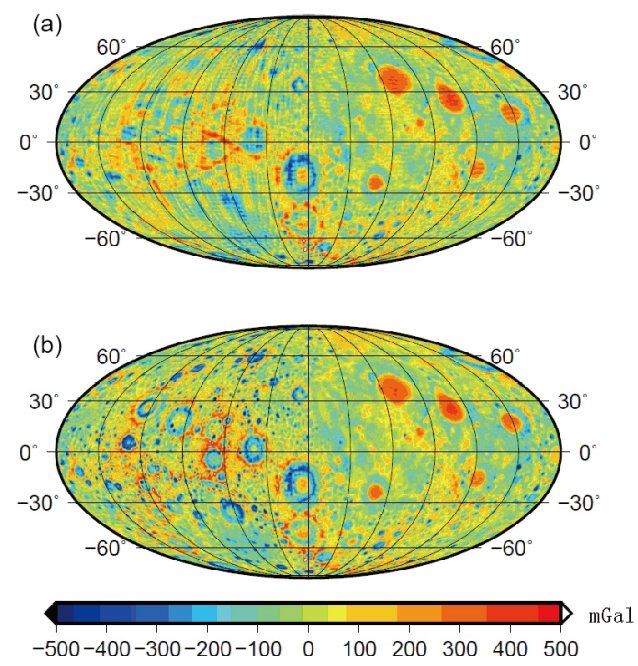

图 1 (网络版彩图)LP165P(a)及GL0420A(b)月球重力场模 型计算的重力异常

Figure 1 (Color online) Free-air gravity anomaly maps from lunar gravity model LP165P (a) and GL0420A (b).

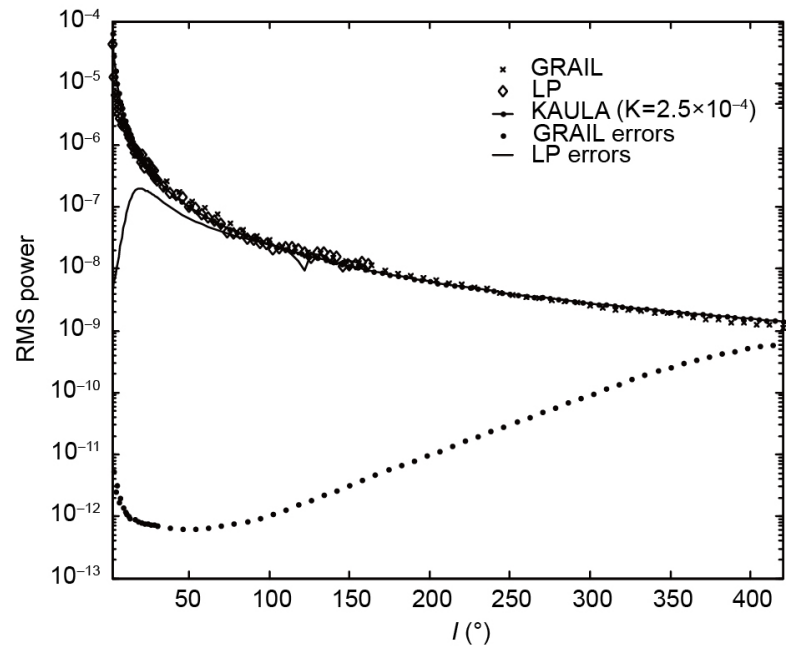

图 2 两模型位系数阶方差及误差阶方差

Figure 2 Spherical harmonics degree variance and error variance.

重力场阶方差曲线, 菱形符号表示LP165P阶方差曲 线; 星型符号表示GL0420A 误差阶方差曲线, 直线表 示LP165P误差阶方差曲线. 根据Zuber等人 ${ }^{[10]}$ 的研 究, 在GL0420A 与LP165P解算中Kaula约束的取值为 $2.5 \times 10^{-4} l^{2}, l$ 为位系数, LP165P模型解算中是全部阶次 用到了 Kaula约束, 但是在GL0420A模型的解算中, 仅 330 以上的阶次施加了约束. 从图2中可以发现, 重力 场模型位系数的阶方差随着阶数的增加而减小, 变化 幅度达几个数量级. 比较GL0420A和LP165P的阶方差 可以发现, 在低阶项上, 各模型一致性很好, 而高阶项 (大概从20阶开始)开始出现偏离. 从误差阶方差上来 看, LP165P重力场模型在20阶上其误差阶方差与阶方 差量级相当, 而GL0420A模型的误差明显降低, 直至 400 阶误差与阶方差量级才相当, 这是因为LP165P缺 乏背面直接观测数据的支持, 而GL0420A模型通过双 星观测的模式, 有效地解决了这一缺陷.

\section{3 “嫦娥”探测器测轨数据处理}

根据我国探月工程“绕、落、回”分步实施的原 则 ${ }^{[22]}$, 目前我国的探月工程已经进行了4次发射任务, 其中 2007 年 10 月 24 日发射的嫦娥一号及 2010 年 10 月 1 日发射的嫦娥二号实现了计划中的“绕月”, 2013年12 月2日发射的嫦娥三号探测器携带了巡视器与着陆器 并成功着陆月球, 实现了计划中的“落月”, 2014年 10 月24日发射的再入返回试验器试验了绕月返回的技 
术. 前 3 次任务中, 探测器均有绕月飞行. 因嫦娥三号 任务采用了发动机喷气进行姿态控制的方式维持卫 星姿态稳定, 段建锋等人 ${ }^{[16]}$ 单独分析了 GRAIL重力场 在频繁姿轨控绕月过程中的应用. 本文为更好地分析 GRAIL重力场对绕月探测卫星的影响, 将主要使用嫦 娥一号及嫦娥二号的绕月测轨数据进行分析.

本文采用动力学方法, 利用 $\mathrm{S}$ 波段微波统一系统 (Unified S Band System, USB)测距、测速和甚长基线 干涉测量(Very Long Baseline Interferometry, VLBI)数 据对嫦娥一号(CE-1)及嫦娥二号(CE-2)进行了精密定 轨, 用于比较两种模型在精密定轨方面的差异. 定轨 过程中选取的力模型如表1所示,包括月球中心引力、 月球非球形摄动、地球中心引力、地球非球形摄动、 第三体引力摄动、太阳光压摄动等 ${ }^{[23]}$. 定轨过程中涉 及的解算参数有卫星的六个初始轨道根数、太阳光 压系数和观测量系统偏差等. 观测数据中USB测距与 测速数据的采样率为 $1 \mathrm{~s}$, VLBI时延和时延率的采样 率约为 $5.2 \mathrm{~s}$.

卫星重叠轨道精度分析可以在一定程度上体现 外符精度. 国内外多数机构在对轨道进行精度评价时 大多采用此方法. 重叠弧段相对于定轨弧段的长度越 短, 其外符精度评价的可信性越高. 本节利用测距和 VLBI测轨数据, 对 $100 \mathrm{~km} \times 100 \mathrm{~km}$ 和 $15 \mathrm{~km} \times 100 \mathrm{~km}$ 的 CE-2环月轨道进行精度分析, 并与CE-1任务相关结果 进行比较, 讨论重力场精度的提高对轨道精度的贡献.

考虑到CE-2轨道的实际情况, 选取没有轨道机动
的弧段每 $18 \mathrm{~h}$ (约9个卫星轨道周期)进行一次轨道确 定, 重叠轨道覆盖弧段长度为 $2 \mathrm{~h}$ (约 1 个卫星轨道周期), 使用的观测数据为事后模式下的VLBI数据联合测距 数据. 对 $100 \mathrm{~km} \times 100 \mathrm{~km}$ 与 $100 \mathrm{~km} \times 15 \mathrm{~km}$ 的环月轨道 进行重叠弧段分析, 结果统计见表2和 3 所示. 表2中 $100 \mathrm{~km} \times 100 \mathrm{~km}$ 环月轨道在同一任务中两种重力场模 型下重叠弧段精度及预报 $18 \mathrm{~h}$ 精度相当, 可见其对重 力场模型并不敏感.

2010年10月26日21时, CE-2开始降轨试验, 变轨至 $100 \mathrm{~km} \times 15 \mathrm{~km}$ 高度的椭圆轨道, 10 月 29 日 10 时卫星抬 升轨道至 $100 \mathrm{~km}$ 高度圆轨道. 变轨试验期间 $100 \mathrm{~km} \times$

表 1 轨道确定策略

Table 1 Orbit determination strategy

\begin{tabular}{cc}
\hline 项 目 & 模 型 \\
\hline 坐标系统 & 月心天球坐标系 \\
月球中心引力 & 质点引力 \\
月球非球形引力场 & LP165P \\
第三体引力 & GL0420A \\
地球中心引力 & 太阳、大行星 \\
地球非球形引力场 & 质点引力 \\
月球潮汐摄动 & JGM-3 \\
太阳光压摄动 & - \\
行星历表 & 固定面质比 \\
数据使用 & DE421 \\
& USB: 测距、测速 \\
\hline
\end{tabular}

表 $2100 \mathrm{~km} \times 100 \mathrm{~km}$ 重叠轨道定轨精度统计

Table 2 Orbit solution statistics for $100 \mathrm{~km} \times 100 \mathrm{~km}$ orbit phase

\begin{tabular}{ccccc}
\hline CE mission & Gravity model & Residuals $1 \sigma(\mathrm{m})$ & Overlap difference $1 \sigma(\mathrm{m})$ & Orbital prediction $1 \sigma(\mathrm{m})$ \\
\hline \multirow{2}{*}{ CE-1 } & LP165P GL0420A & 17.0033 & 418.98 & 557.6 \\
& & 16.1056 & 418.20 & 551.3 \\
\multirow{2}{*}{ CE-2 } & LP165P GL0420A & 5.2946 & 69.33 & 57.6 \\
& & 5.1652 & 43.65 & 51.3 \\
\hline
\end{tabular}

表 $3100 \mathrm{~km} \times 15 \mathrm{~km}$ 重叠轨道定轨精度统计

Table 3 Orbit solution statistics for $100 \mathrm{~km} \times 15 \mathrm{~km}$ orbit phase

\begin{tabular}{ccccc}
\hline CE mission & Gravity model & Residuals $1 \sigma(\mathrm{m})$ & Overlap difference $1 \sigma(\mathrm{m})$ & Orbital prediction $1 \sigma(\mathrm{m})$ \\
\hline \multirow{2}{*}{ CE-1 } & \multirow{2}{*}{ LP165P GL0420A } & 20.00 & 325.1165 & 508.1 \\
& & 6.73 & 179.1052 & 274.5 \\
\multirow{2}{*}{ CE-2 } & \multirow{2}{*}{ LP165P GL0420A } & 18.81 & 142.98 & 108.1 \\
& & 6.48 & 56.78 & 72.0 \\
\hline
\end{tabular}


$15 \mathrm{~km}$ 轨道状态大约维持了 $60 \mathrm{~h}$. 整个降轨过程 CE-2 处于非通视状态(Edge-On $)^{[3]}$, 即每圈CE-2 都有部分时 间处于月球背面, 月球背面的重力异常将会对卫星轨 道产生更大的影响. 由表3中重叠弧段、18 h 预报精 度统计值可以看出, 相比于 CE-1卫星, CE-2卫星此阶 段对月球背面的高阶重力场信息更为敏感, 测轨数据 能够更好地反映重力场信号. 因CE-2任务中使用了新 的测量设备及数据处理方法, 显著提高了观测精度 ${ }^{[24]}$. 测量数据精度对定轨精度有重要影响. 因此从表 3 中 可以看出, 两种月球重力场模型下, CE-2任务的重叠 弧段精度及预报 $18 \mathrm{~h}$ 精度均优于CE-1任务.

\section{4 结论}

本文通过对不同高阶重力场模型 LP165P和 GL0420A的物理特征以及定轨差异进行了分析, 得出 如下结论: (1) 由于 GRAIL任务直接提取了远月面的
重力场信息, 重力场模型GL0420A的功率谱在远月面 的中高阶次明显高于LP165P. (2) 两个重力场模型在近 月面自由空气重力异常的分布相近, 而在远月面存在 明显的差异, 其中 GL0420A的自由空气重力异常在远 月面的分辨率高于LP165P. 其中GL0420A 能够分辨出 远月面小型撞击盆地水准面的特征, 这说明GL0420A 适合于作远月面地球物理参数的估计等. (3) 对 CE-1 及 CE-2卫星的测轨数据使用不同的重力场模型进 行轨道解算比较. 计算表明, 对于 $100 \mathrm{~km} \times 100 \mathrm{~km}$, $100 \mathrm{~km} \times 15 \mathrm{~km}$ 轨道阶段, 由于 GL0420A包含了远月 面真实的重力场信息, 使用 GL0420A重力场模型比 LP165P重力场模型的轨道解算结果好, 对于 $100 \mathrm{~km}$ $\times 15 \mathrm{~km}$ 月球轨道, 高阶重力场能显著提高定轨精度, GL0420A更适用于低轨月球探测器的精密定轨计算. 本文采用多种方式分析了两个重力场模型的差异, 为 我国后续探月卫星精密定轨在重力场选择上提供了 参考.

\section{参考文献}

1 Kaula W M. The gravitational field of the moon. Science, 1969, 166: 1581-1588

2 Williams J G, Boggs D H. Lunar core and mantle, what does LLR see. In: Proceedings of the 16th International Workshop on Laser Ranging. Poznan: ResearchGate, 2008. 101-120

3 Cao J F, Hu S J, Liu L, et al. A comparative study on the accuracy of lunar gravity field models (in Chinese). J Spacecr TT\&C Tech, 2012, 31: 74-79 [曹建峰, 胡松杰, 刘否, 等. 月球重力场模型精度比较研究. 飞行器测控学报, 2012, 31: 74-79]

4 Konopliv A S, Binder A B, Hood L L, et al. Improved gravity field of the moon from lunar prospector. Science, 1998, 281: 1476-1480

5 Konopliv A S, Asmar S W, Carranza E, et al. Recent gravity models as a result of the lunar prospector mission. Icarus, 2001, 150: 1-18

6 Binder A B. Lunar prospector: Overview. Science, 1998, 281: 1475-1476

7 Yan J G, Li F, Ping J S. Precision orbit determination of lunar spacecraft LP and lunar gravity field model solution (in Chinese). J Astronaut, 2011, 32: 767-774 [鄢建国, 李斐, 平劲松. 月球探测器LP精密定轨及月球重力场模型解算. 宇航学报, 2011, 32: 767-774]

8 Wei Z, Lu X L, Houtse H, et al. Simulation of the Earth's gravitational field recovery from GRACE using the energy balance approach. Prog Nat Sc, 2005, 15: 596-601

9 Zheng W, Xu H Z, Zhong M, et al. Progress in international lunar exploration programs (in Chinese). Prog Geophys. 2012, 27: 2296-2307 [郑 伟, 许厚泽, 钟敏, 等. 月球探测计划研究进展. 地球物理学进展, 2012, 27: 2296-2307]

10 Zuber M T, Smith D E, Watkins M M, et al. Gravity field of the moon from the gravity recovery and interior laboratory (GRAIL) mission. Science, 2013, 339: 668-671

11 Lemoine F G, Goossens S, Sabaka T J, et al. High-degree gravity models from GRAIL primary mission data. J Geophys Res Planets, 2013, 118: 1676-1698

12 Lemoine F G, Goossens S, Sabaka T J, et al. GRGM900C: A degree 900 lunar gravity model from GRAIL primary and extended mission data. Geophys Res Lett, 2014, 41: 3382-3389

13 Konopliv A S, Park R S, Yuan D N, et al. The JPL lunar gravity field to spherical harmonic degree 660 from the GRAIL primary mission. J Geophys Res Planets, 2013, 118: 1415-1434

14 Konopliv A S, Park R S, Yuan D N, et al. High-resolution lunar gravity fields from the GRAIL primary and extended missions. Geophys Res Lett, 2014, 41: 1452-1458

15 Yan J, Goossens S, Matsumoto K, et al. CEGM02: An improved lunar gravity model using Chang'E-1 orbital tracking data. Planet Space Sci, 
2012, 62: 1-9

Duan J F, Zhang Y, Chen M, et al. Application of GRAIL lunar gravity field model in attitude and orbit control for CE-3 satellite (in Chinese). J Spacecr TT\&C Tech, 2014, 33: 342-347 [段建锋, 张宇, 陈明, 等. 嫦娥三号姿轨控过程中GRAIL重力场模型的应用. 飞行器测控学报, 2014, 33: 342-347]

17 Ye M, Li F, Yan J G, et al. Orbit determination ability analysis of the GRAIL gravity model (in Chinese). Geomatics Inf Sci Wuhan Univ, 2016, 41: 93-99 [叶茂, 李斐, 滒建国, 等. GRAIL月球重力场模型定轨性能分析. 武汉大学学报信息科学版, 2016, 41: 93-99]

18 Hu S J, Tang G S. BACC orbit determination and analysis software for deep-space explorers (in Chinese). J Spacecr TT\&C Tech, 2010, 29: 69-74 [胡杰松, 唐歌实. 北京中心深空探测器精密定轨与分析软件系统. 飞行器测控学报, 2010, 29: 69-74]

19 Guo J Y. Fundamentals of Geophysics (in Chinese). Beijing: Surveying and Mapping Publishing House, 2001: 178-182 [郭俊义. 地球物理学 基础. 北京: 测绘出版社, 2001：178-182]

20 Wieczorek M A. Gravity and topography of the terrestrial planets. Treatise Geophys, 2007, 10: 165-206

21 Kaula W M. Theory of Satellite Geodesy: Applications of Satellites to Geodesy. New York: W. H. Courier Dover Publications, 2000

22 Ouyang Z Y. Scientific objectives of Chinese lunar exploration project and development strategy (in Chinese). Adv Earth Sci, 2004, 19: 351-358 [欧阳自远. 我国月球探测的总体科学目标与发展战略. 地球科学进展, 2004, 19: 351-358]

23 Duan J F, Cao J F, Zhang Y, et al. An effective orbit determination strategy to improve the cohesion precision of ChangE' one satellite's precise ephemeris before and after unloading (in Chinese). Aerospace Control, 2015, 33: 66-71 [段建锋, 曹建峰, 张宇, 等. 一种有效提高CE-1卫星 卸载前后精密星历衔接精度的定轨策略. 航天控制, 2015, 33: 66-71]

\title{
The improvements of Chang' $E$ series satellites orbit determination from GRAIL lunar gravity model
}

\author{
DUAN JianFeng ${ }^{1,2^{*}}$, CAO JianFeng ${ }^{1,3}$, CHEN Ming ${ }^{1} \&$ ZHANG Yu ${ }^{1}$ \\ ${ }^{1}$ Beijing Aerospace Control Center, Beijing 100094, China; \\ ${ }^{2}$ School of Aerospace, Tsinghua University, Beijing 100083, China; \\ ${ }^{3}$ Science and Technology on Aerospace Flight Dynamics Laboratory, Beijing 100094, China
}

Here we analyze different features between the gravity models of GL0420A and LP165P, including the cross-power spectra of gravity and lunar free-air gravity anomaly. The results show that GL0420A has greatly improved the order and the accuracy of the lunar gravity model, and GL0420A can better reveal the characteristics of the moon. Orbit determination for Chang'E series lunar probe at $100 \mathrm{~km} \times 100 \mathrm{~km}$ and $100 \mathrm{~km} \times 15 \mathrm{~km}$ orbit phases is performed with different gravity field models. The results show a better orbit solution with $10 \mathrm{~m}$-level improvement that can be obtained using GL0420A and the solution is more suitable for high accuracy orbit determination for lunar probes.

Chang'E series lunar probe, GL0420A lunar gravity model, LP165P lunar gravity model, accuracy analysis

PACS: 95.55.Pe, 91.10.Fc, 95.40.+s, 91.10.Op

doi: $10.1360 /$ SSPMA2016-00320 